\title{
Negative and Positive Reappraisal After a Romantic Break-Up
}

\author{
Scarlett B. Horner ${ }^{\mathrm{a}, \mathrm{b}}$ \& Sandra J. E. Langeslaga
}

This study compared negative reappraisal of an ex-partner and positive reappraisal of a situation after a break-up. Negative reappraisal was expected to reduce love, increase unpleasantness, reduce upsetness about the break-up, and reduce motivated attention to the ex-partner as measured by the late positive potential (LPP) compared to positive reappraisal. In this study, twentyfour participants who were upset about a break-up viewed pictures of their ex-partner in two reappraisal conditions and a no reappraisal condition. In the negative reappraisal condition, participants thought about negative aspects of their ex-partner. In the positive reappraisal condition, participants thought about positive aspects of the situation. Subsequently, participants viewed expartner pictures and the LPP was measured. Participants rated infatuation, attachment, valence, and upsetness about the break-up. Even though numerical differences were in line with our hypotheses, we found no evidence of significant differences between conditions for infatuation, attachment, valence, upsetness, or LPP amplitude in the preregistered analyses.

Key words: love, reappraisal, regulation, event-related potentials (ERP), late positive potential (LPP)

Anyone who has gone through a romantic break-up knows how upsetting it can be. At its worst, heartbreak can cause insomnia, depression, and intrusive thoughts (Field, 2011). A break-up can also cause a loss of self-concept in some people (Mason, Law, Bryan, Portley, \& Sbarra, 2012; Slotter, Gardner, \& Finkel, 2010). Because of the negative consequences of a break-up, it would be beneficial for someone to effectively get over a break-up.

Emotion regulation is the ability to change or maintain positive and negative emotions (Gross, 1998). One of the ways people can regulate their emotions is through cognitive reappraisal or re-interpreting a situation. There are many methods of emotion regulation, but reappraisal has been found to be an effective method of emotion regulation (Gross, 2002; Nezlek \& Kuppens, 2008). In particular, two previous studies have shown that negative reappraisal of the ex-partner, the relationship, or the future of the relationship decreased love feelings (Langeslag \& Sanchez, 2018; Langeslag \& Van Strien, 2016). This is an important finding, but love feelings can be broken down further. For example, two types of love are infatuation and attachment. Infatuation is defined as a very strong attraction towards another person, while attachment is defined as an emotional bond with another person (Fisher, 1998). In a previous study, both infatuation and attachment decreased compared to no regulation when participants downregulated their love feelings using negative reappraisal of the (ex-)partner (Langeslag \& Van Strien, 2016). Unfortunately, while negative reappraisal of the (ex-)partner made people feel less in love, it also caused participants to feel more unpleasant (Langeslag \& Sanchez, 2018; Langeslag \& Van Strien, 2016). It is as of yet unclear, however, whether negative reappraisal decreased how upset people were about the break-up, as the previous studies did not measure this important outcome variable. Generally, love feelings after a break-up are associated with sadness (Sbarra \& Ferrer, 2006) and decreased well-being (Mason et al., 2012). As sadness and well-being are related to love feelings after a break-up, it is possible that decreasing love feelings decreases upset feelings related to the break-up. If people are less upset about the break-up after negative reappraisal, that may be an advantageous strategy.

However, if negative reappraisal of the ex-partner does make people feel more unpleasant, a different strategy may be needed to increase pleasant feelings, such as positive reappraisal of the situation. Positive reappraisal entails focusing on positive aspects of a situation. In one study, participants were able to use positive reappraisal to increase amusement while watching a video (Giuliani, McRae, \& Gross, 2008). This supports the idea that positive reappraisal while looking at stimuli can increase positive emotion. In another study, participants were instructed to think of positive outcomes and how things get better in response to a negative stimulus, which led participants to feel more pleasant (Ochsner et al., 2004). Positive reappraisal is also effective at improving well-being in negative situations. For example, women with breast cancer who reported performing positive reappraisal more often had greater perceived health, as well as posttraumatic growth after the breast cancer subsided (Sears, Stanton, \& Danoff-Burg, 2003). Therefore, positive reappraisal of the situation may be an effective regulation strategy to cope with heartbreak, but this specifically has not been tested yet.

The current study compared negative reappraisal of the ex-partner and positive reappraisal of the situation. The first research question was if negative reappraisal of the ex-partner and positive reappraisal of the situation differentially modulate love feelings. Because negative reappraisal of the ex-partner focuses on the object of the love feelings while positive reappraisal of the situation does not, we expected that negative reappraisal of the ex-partner would decrease love feelings, such as infatuation and attachment, more than positive reappraisal of the situation. The second research question was if negative reappraisal of the ex-partner and positive reappraisal of the situation differentially modulate the valence of affect. Because previous studies have shown that negative reappraisal of the ex-partner makes people feel more unpleasant (Langeslag \& Sanchez, 2018; Langeslag \& Van Strien, 2016) and that positive reappraisal of the situation makes people feel more pleasant (Kanske, Heissler, Schönfelder, Bongers, \& Wessa, 2011; Ochsner et al., 2004; Sears et al., 2003), we expected that positive reappraisal of the situation would make people feel more pleasant than negative reappraisal of the ex-partner. The third research question was if negative reappraisal of the expartner and positive reappraisal of the situation differentially modulate how upset people feel about a romantic break-up. Because negative reappraisal of the ex-partner is expected to 
reduce love feelings more than positive reappraisal of the situation (see the first hypothesis above) and because remaining love feelings for the ex-partner have been associated with reduced recovery from the break-up (Mason et al., 2012; Sbarra \& Ferrer, 2006), we expected that negative reappraisal of the ex-partner would make people feel less upset about the break-up than positive reappraisal of the situation.

Another way to measure the effectiveness of certain emotion regulation methods is to measure attention given to regulated stimuli. Emotional stimuli are given attentional priority over neutral stimuli (Compton, 2003). One way of measuring attention is by using the late positive potential (LPP), a component of the event-related potential (ERP) that is positive, maximal over the medial-parietal scalp, and begins approximately $300-400 \mathrm{~ms}$ after stimulus onset, that can be used to measure attention. The LPP is enhanced in response to target stimuli, emotional stimuli, and love stimuli (Langeslag, Jansma, Franken, \& Van Strien, 2007; Schupp, Flaisch, Stockburger, \& Junghöfer, 2006). The more arousing a stimulus is, the larger the LPP amplitude (Hajcak, Macnamara, \& Olvet, 2010; Schupp et al., 2006), and the larger the LPP amplitude is, the more someone is attending to a stimulus. In one study, pictures of the beloved elicited a greater LPP amplitude compared to pictures of opposite-sex friends and strangers, suggesting people pay greater attention to the beloved than friends and strangers (Langeslag et al., 2007). Another study showed that the LPP amplitude in response to emotionally charged pictures was smaller when participants performed reappraisal than when participants passively viewed the picture (Hajcak \& Nieuwenhuis, 2006), which supports the idea that emotion down-regulation reduces the LPP amplitude. Reappraisal also change the LPP amplitude while looking at pictures of the beloved. In one study, positive reappraisal of the beloved increased the LPP amplitude while negative reappraisal of the beloved decreased the LPP amplitude compared to passive viewing (Langeslag \& Van Strien, 2016). In another previous study, negative reappraisal of the expartner decreased the LPP in response to pictures of the expartner (Langeslag \& Sanchez, 2018).

The final research question was if negative reappraisal of the ex-partner and positive reappraisal of the situation differentially modulate motivated attention for the ex-partner, as indicated by the LPP amplitude. Because negative reappraisal of the ex-partner focuses on the ex-partner while positive reappraisal of the situation does not, we expected that negative reappraisal of the ex-partner would reduce the LPP amplitude in response to a picture of that ex-partner more than positive reappraisal of the situation.

This study was preregistered with the Preregistration Challenge of the Center for Open Science (https://osf.io/3ngvu/). That means that the research questions, hypotheses, design, and analyses were determined before the start of the data collection. Preregistration ensures that the study is confirmatory rather than exploratory. On the preregistration form, we called our conditions love regulation, emotion regulation, and no regulation instead of negative reappraisal of the ex-partner, positive reappraisal of the situation, and no reappraisal respectively. We have changed the names in this manuscript because the new names describe the participant instructions rather than the hypothesized outcomes of the strategies, which makes it easier for the reader to parse the information. Please note that other than the names of the two regulation conditions, our research questions, hypotheses, and analyses were the same as on the preregistration form.

\section{Methods \\ Participants}

Unfortunately, there currently is no convenient way to perform a power analysis with more than one within-subject variable in G*Power $\left(\mathrm{G}^{*}\right.$ Power Feedback, personal communication, January 9, 2018). Instead, we determined the sample size on the basis of the previous study (Langeslag \& Sanchez, 2018) that had 24 participants. For this study, 27 participants were recruited from the University of Missouri St. Louis (UMSL), Craigslist, ResearchMatch, and the greater St. Louis area. All participants had experienced a break-up and were upset about it at the time of the study, as indicated by a score of two or higher on the question "how upset are you about the break-up?" (see below). Other inclusion criteria included having normal or corrected-to-normal vision, having no psychiatric or mental disorders, and not using medications that affect the central nervous system. Three participants had to be excluded from the analysis, one because of a computer error, one for excessive artifacts (see below), and one for not having normal or corrected-to-normal vision, leaving 24 participants (16 women) ranging from ages $28-38$ years $(\mathrm{M}=27.21, \mathrm{SD}=$ 6.18 ) with usable data. Twenty participants were right-handed, two participants were left-handed, and two participants were ambidextrous as determined by the handedness questionnaire (see below). The study was approved by the University of Missouri-St. Louis institutional review board. Participants provided written informed consent before participating in the study. Participants who were students in psychology courses at UMSL that offered (extra) credit for research participation could choose to receive either course credit or $\$ 40$ for their participation in the study. Two participants chose course credit. Participants who were not students in those specific courses received $\$ 40$ for their participation in the study.

Stimuli

Participants provided 28 non-explicit, non-intimate pictures of their ex-partner. The pictures showed the ex-partner partially obscured or unobscured, in a variety of situations, and were allowed to contain other people, as was done in a previous study (Langeslag \& Sanchez, 2018). These pictures mimicked situations in which the participant could encounter their expartner. Please note that picture content did not confound the regulation effects, because each picture was shown in each regulation condition.

\section{Procedure}

Participants completed a general questionnaire about the break-up (Langeslag \& Sanchez, 2018). Participants reported the gender of their ex-partner, how long the relationship had lasted, the status of the relationship (married, cohabiting, or not cohabiting), how good the relationship with their ex-partner was $(1=$ very bad, 9 = very good $)$, how long ago the relationship ended, who ended the relationship (participant, expartner, both), and how upset they were about the break-up (1 $=$ not upset at all, $9=$ very upset). Participants then completed the Infatuation and Attachment Scale (IAS) (Langeslag, Muris, \& Franken, 2013), which measures how infatuated and attached participants were to their ex-partners on a seven-point scale. Participants also completed a handedness questionnaire (Bryden, 1985). 
Then, the electrode cap was attached, and participants completed a regulation task while their electroencephalogram (EEG) was recorded. The regulation task had three conditions: negative reappraisal of the ex-partner, positive reappraisal of the situation, and no reappraisal. As shown in Figure 1, each trial started with a prompt (See Appendix A for list of prompts) for five seconds, followed by a fixation cross with a jittered duration of 500-700 ms, a picture for one second, and finally a blank screen for one second. In the negative reappraisal condition, the prompts were questions about negative traits of the ex-partner (e.g. "What is an annoying habit of your ex?") and participants were instructed to silently answer the question. In the positive reappraisal condition, the prompts were questions about positive aspects of the situation (e.g. "What positive change can you make now that you're single?") and participants were instructed to silently answer the question. There were 28 prompts in the negative reappraisal condition and 28 prompts in the positive reappraisal condition. Every prompt was used once. In the no reappraisal condition, participants saw asterisks instead of prompts and were not instructed to think of anything in particular. After the prompts/asterisks and the fixation cross, participants passively viewed a picture of the ex-partner. Participants were asked to limit movements and to try not to blink during the presentation of the fixation cross and the picture. Condition was blocked and the order of the three blocks was counterbalanced between participants. Each block contained 28 trials. At the end of each block, participants completed ratings of infatuation, attachment, valence, and upsetness about the break-up by using a slider. Participants were asked "How infatuated do you feel?", "How attached do you feel?", "How negative or positive do you feel?", and "How upset about the break-up do you feel?" For ratings of infatuation, attachment, and upsetness about the break-up, the left side was labeled "not at all" and the right side was labeled "very much." For the valence rating, the left side was labeled "negative" and the right side was labeled "positive."

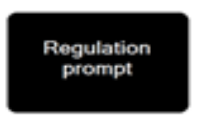

5 seconds

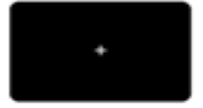

$0.5-0.7$ seconds

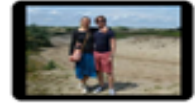

1 second

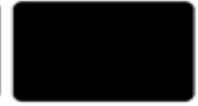

1 second $\times 28$

Fig. 1 Trial Overview and End of Block Ratings

Before completing the task, participants completed a practice block that consisted of one trial of each condition and included prompts not used in the main task. The images in the practice block were images unrelated to the ex-partner. After the task was completed, the EEG cap was removed and participants were debriefed, thanked, and compensated.

Electroencephalogram (EEG) recording and signal processing

The EEG was recorded using a 32 channel amplifier and data acquisition software (ActiveTwo System, BioSemi). The $32 \mathrm{Ag}-\mathrm{AgCl}$ active electrodes were connected to the scalp through a head cap (BioSemi) according to the 10-20 International System (Fp1/2, AF3/4, Fz, F3/ 4, F7/8, FC1/2, FC5/6, Cz, C3/4, T7/8, CP1/2, CP5/6, Pz, P3/4, P7/8, PO3/4,

Oz, O1/2). Vertical electrooculogram (VEOG) and horizontal electrooculogram (HEOG) was recorded by attaching electrodes (UltraFlat Active electrodes, BioSemi) above and below the left eye, and at the outer canthi of both eyes. Two electrodes were also placed at the at the left and right mastoids (M1/2). An active electrode (CMS - common mode sense) and a passive electrode (DRL - driven right leg) were used to create a feedback loop. Signals were digitized using a sampling rate of $512 \mathrm{~Hz}$, a 24-bit A/D conversion, and a low-pass filter of $134 \mathrm{~Hz}$.

Data were analyzed with BrainVision Analyzer 2 (Brain Products, Gilching, Germany). A maximum of one bad electrode per participant was corrected using spherical spline topographic interpolation. Offline, an average mastoids reference was applied because that is the preferred reference to study the emotional modulation of the LPP (Hajcak, Weinberg, Macnamara, \& Foti, 2011). The data were filtered using a 0.10$30 \mathrm{~Hz}$ band pass filter (phase shift-free Butterworth filters; 24 $\mathrm{dB} /$ octave slope) and a $60 \mathrm{~Hz}$ notch filter. Data were segmented starting $200 \mathrm{~ms}$ before the onset of the ex-partner picture and ending $1000 \mathrm{~ms}$ after the picture's onset. The mean $200 \mathrm{~ms}$ before picture onset was used for baseline correction. Ocular artifact correction was applied semi-automatically (Gratton, Coles, \& Donchin, 1983). Artifact rejection was performed at individual electrodes with a baseline-to-peak minimum and maximum criterion of -75 to $+75 \mu \mathrm{V}$. To obtain reliable emotional modulation of the LPP, participants must have at least 12 accepted trials per condition (Moran, Jendrusina, \& Moser, 2013), so as mentioned above, two participants with fewer than 12 trials per electrode per condition were excluded from analyses. Average waveforms were computed per condition. At the electrodes used in the analyses (see below), the average number of accepted trials per condition ranged from 27.3 to 28.0 out of 28 .

\section{Statistical Analyses}

Infatuation, attachment, valence, and upsetness about break-up ratings were transformed from the slider to a scale of 0-100 (0 being far left, 100 being far right), and tested using a

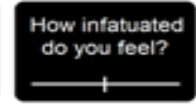

Until response

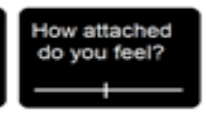

Until response

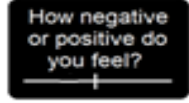

Until response

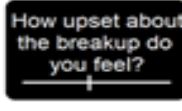

Until response repeated measures analysis of variances (rmANOVA) with the factor Condition (negative reappraisal of the ex-partner, positive reappraisal of the situation, no reappraisal). The LPP in response to the ex-partner picture was quantified by a mean amplitude measure in a 400-1,000 ms time window (Langeslag \& Sanchez, 2018). Mean amplitude measures at electrodes F3, $\mathrm{Fz}, \mathrm{F} 4, \mathrm{C} 3, \mathrm{Cz}, \mathrm{C} 4, \mathrm{P} 3, \mathrm{Pz}$, and P4 for each condition were submitted to a rmANOVA with factors Condition, Caudality (frontal, central, parietal), and Laterality (left, center, right). Only significant effects involving factor Condition are reported because those are relevant to the research questions.

Degrees of freedom (df) were corrected with the Greenhouse-Geisser correction. The $F$ values, uncorrected $d f \mathrm{~s}$, the epsilon $(\varepsilon)$ values, corrected probability levels, and effect 
sizes $\left(\eta_{\mathrm{p}}{ }^{2}\right)$ are reported. A two-sided alpha of less than 0.05 was considered statistically significant. Type I error rate was controlled for by Fisher's least significant difference (LSD) procedure, which only conducted follow-up tests for significant main and interaction effects (i.e., omnibus tests). Follow up tests used to compare the three conditions with each other were paired samples t-tests. Effect size was assessed using Cohen's $d^{\prime}$.

\section{Results \\ Participant Characteristics}

Twenty-one participants had an opposite-sex ex-partner and three male participants had a same-sex ex-partner. The average length of the relationship was 40.92 months (range: 11144 months). Three participants had been married, nine had been cohabiting, 11 had not been cohabiting, and one did not wish to answer. On average, the quality of the relationships was rated 6.46 out of 9 (range: 4-9 out of 9). On average, the relationships ended 14.42 months ago (range: 1-132 months). Ten participants had initiated the breakup, eight participants were broken up with by the ex-partner, and six participants said both parties ended the relationship. On average, participants rated their upsetness about the break-up 7.17 out of 9 (range: 3-9, which shows that all participants were upset about the break-up to some degree). Please note that the heterogeneity of the sample does not confound the regulation effects, because each participant completed every regulation condition (i.e., the study has a within-subject design).

The average IAS infatuation score was 4.35 out of 7 (range: 2.0-6.3), and the average IAS attachment score was 3.39 out of 7 (range: 1.4-6.3). Exploratory analyses showed that there tended to be a positive association between IAS infatuation scores and how upset about the break-up participants were, $r=.373, p=.073$, and that there was no correlation between IAS attachment scores and how upset about the break-up participants were, $r=.091, p=.671$.

\section{Ratings - Preregistered analyses}

See table 1 and figure 2 for the ratings data in each condition. Even though infatuation ratings were numerically lowest after negative reappraisal of the ex-partner, intermediate after positive reappraisal of the situation, and highest after no reappraisal, the main effect of Condition was not significant, $F(2,46)=1.178, \varepsilon=.781, p=.309, \eta_{\mathrm{p}}^{2}=.049$. Likewise, attachment ratings were numerically lowest after negative reappraisal of the ex-partner, intermediate after positive reappraisal of the situation, and highest after no reappraisal, and the main effect of Condition trended towards significance, $F(2,46)=3.544, \varepsilon=.678, p=.057, \eta_{\mathrm{p}}^{2}=.134$. In addition, participants felt most pleasant after positive reappraisal of the situation, intermediately pleasant after no reappraisal, and least pleasant after negative reappraisal of the ex-partner, and the main effect of Condition trended towards significance, $F(2,46)$ $=3.748, \varepsilon=.671, p=.051, \eta_{\mathrm{p}}{ }^{2}=.140$. Finally, ratings of upsetness about the break-up were very similar across conditions, and the main effect of Condition was not significant, $F(2,46)<1$, ns.
Table 1 Average ratings (standard deviations in parentheses) of infatuation, attachment, valence, and upsetness about the break-up

\begin{tabular}{|c|c|c|c|}
\hline Condition & Negative Reapprai sal & Positive Reappraisal & No Reappraisal \\
\hline Infatuation Rating & $42.92(30.23)$ & $45.92(28.72)$ & $50.33(30.27)$ \\
\hline Attachment Rating & $43.92(30.18)$ & $50.50(28.83)$ & $58.92(27.55)$ \\
\hline Valence Rating & $36.54(26.62)$ & $50.04(28.88)$ & $42.63(23.33)$ \\
\hline Upsetness Rating & $62.54(24.97)$ & $61.79(25.89)$ & $64.38(25.52)$ \\
\hline
\end{tabular}

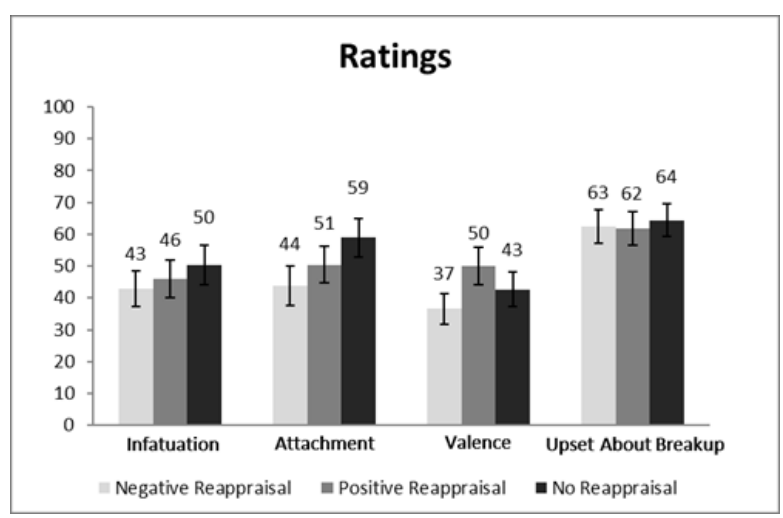

Fig. 2 Average ratings of infatuation, attachment, valence, and upsetness about the break-up. Error bars indicate standard error of the mean.

\section{Ratings - Exploratory Analyses}

We performed exploratory analyses of individual differences related to gender, relationship status, and relationship duration, although the different groups were very small. In rmANOVAs with the within-subject factor Condition and the between-subject factor Gender (woman, man) on the infatuation, attachment, valence, and upsetness ratings there were no significant interactions between Condition and Gender, all $F_{\mathrm{s}}(2,44)<2.996$, all $p \mathrm{~s}>.074$. In rmANOVAs with the within-subject factor Condition and the betweensubject factor Relationship Status (married, cohabiting, not cohabiting) on the infatuation, attachment, valence, and upsetness ratings, there were no significant interactions between Condition and Relationship Status, all $F_{\mathrm{s}}(2,22)<1$, $n s$. In repeated measures analyses of covariance (rmANCOVAs) with within-subject factor Condition and continuous predictor Relationship Duration on the infatuation, attachment, valence, and upsetness ratings there were no significant interactions between Condition and Relationship Duration, all $F_{\mathrm{s}}(2,44)<2.586$, all $p s>.107$.

\section{ERP-Preregistered analyses}

See figure 3, figure 4, and table 2 for the LPP amplitude at $\mathrm{Fz}, \mathrm{Cz}$, and $\mathrm{Pz}$ and the scalp topography of the regulation effects. As can be seen, the ERP in response to pictures of the ex-partner was less positive after negative reappraisal of the expartner and positive reappraisal of the situation than after no reappraisal at electrode $\mathrm{Pz}$ from around $200 \mathrm{~ms}$. However, none of the effects involving the factor Condition were significant, all $F$ s $<1.333$, all $p$ s $>.220$. 
Research Article (2019)

Table 2 Average LPP amplitude in the 400-1000 ms time window in microvolts (standard deviation in parentheses) at the nine electrodes included in the analyses in the three conditions.

\begin{tabular}{rl|lll}
\multicolumn{1}{c}{ Condition } & Negative Reapprai sal & Positive Reappraisal & No Reappraisal \\
\hline F3 & $1.75(5.87)$ & $1.30(5.47)$ & $2.28(5.27)$ \\
Fz & $2.24(6.13)$ & $1.91(5.25)$ & $3.09(4.85)$ \\
F4 & $2.49(5.63)$ & $1.19(4.87)$ & $3.47(5.37)$ \\
C3 & $2.73(5.81)$ & $2.78(4.48)$ & $3.69(4.45)$ \\
Cz & $2.55(5.91)$ & $2.80(4.78)$ & $2.75(6.08)$ \\
C4 & $3.14(4.91)$ & $2.35(4.70)$ & $4.46(4.44)$ \\
P3 & $4.01(4.64)$ & $4.79(4.21)$ & $5.80(3.48)$ \\
Pz & $2.92(3.95)$ & $3.68(3.81)$ & $5.45(3.28)$ \\
P4 & $4.41(3.61)$ & $4.04(4.70)$ & $5.52(3.67)$
\end{tabular}
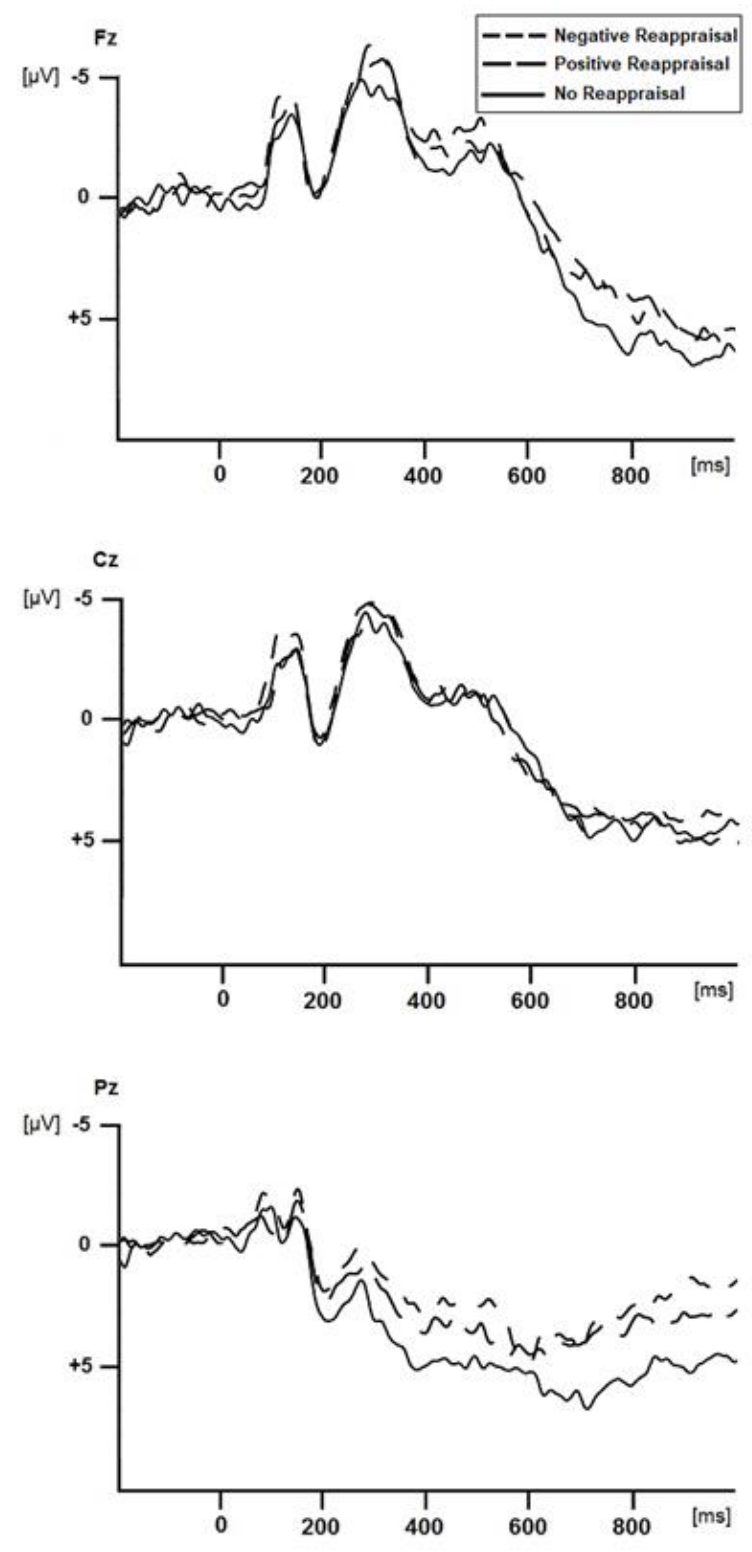

Fig. 3 Event-related potentials at electrodes $\mathrm{Fz}, \mathrm{Cz}$, and Pz. Positive is plotted downwards 

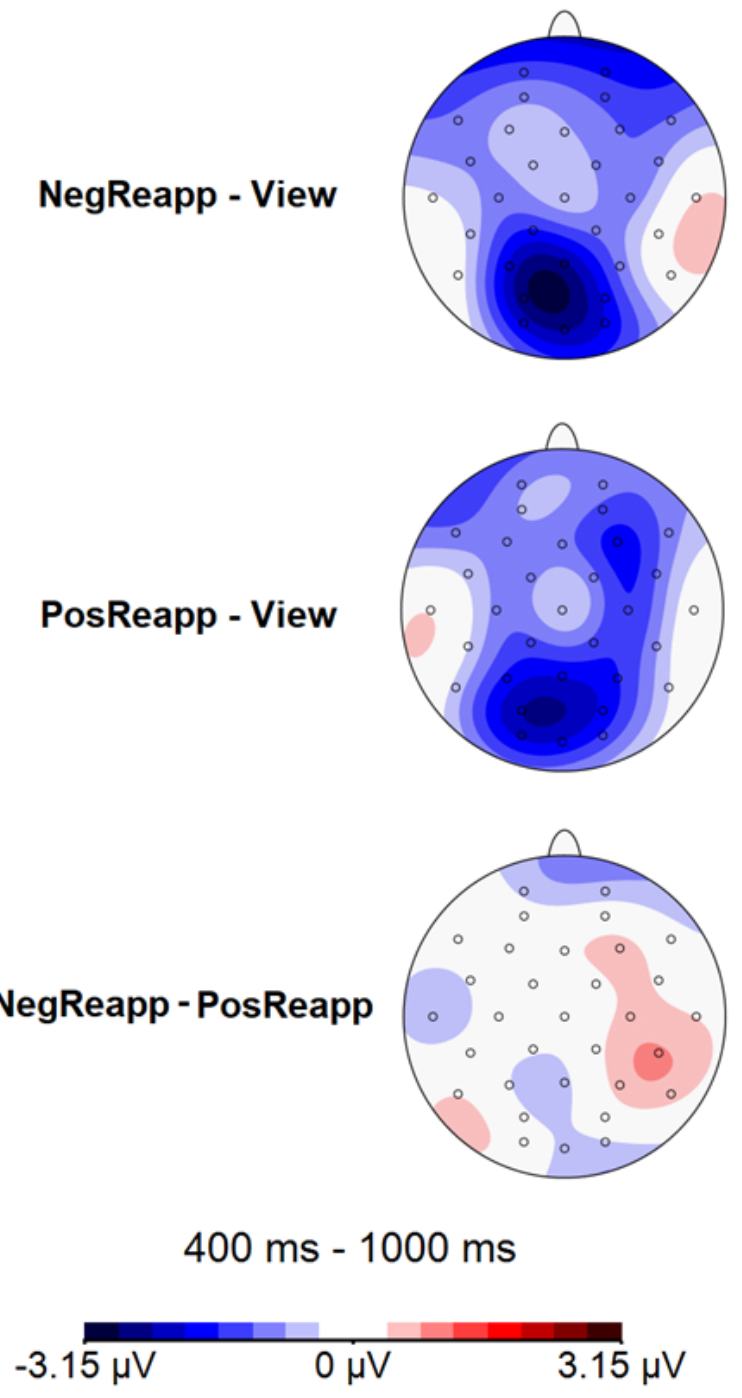

Fig. 4 Scalp Topography of the Regulation Effects

\section{ERP - Exploratory Analyses}

Because visual inspection of the data revealed that the largest difference between conditions occurred around electrode Pz (see Figs. 3 and 4), which is where the LPP is typically maximal (Hajcak et al., 2010), we performed exploratory analyses using a rmANOVA with factor Condition on the ERP amplitude at electrode $\mathrm{Pz}$ alone. At electrode $\mathrm{Pz}$, there was a main effect of Condition, $F(2,46)=4.247, p=$ $.021, \eta_{\mathrm{p}}{ }^{2}=.156$. Follow up tests showed that the LPP amplitude in response to pictures of the ex-partner was less positive after negative reappraisal of the ex-partner $(\mathrm{M}=2.92, \mathrm{SD}=3.95)$ than after no reappraisal $(\mathrm{M}=5.45, \mathrm{SD}=3.28), p=.006$, Cohen's $d^{\prime}=.697$. In addition, the LPP in response to pictures of the ex-partner was less positive after positive reappraisal of the situation $(M=3.68, \mathrm{SD}=3.81)$ than after no reappraisal, but this difference only trended towards significance, $p=.075$, Cohen's $d$ ' $=.498$. There was no significant difference in LPP amplitude at $\mathrm{Pz}$ between negative reappraisal of the ex-partner and positive reappraisal of the situation, $p=.399$, Cohen's $d$ ' $=.196$.

\section{Discussion}

Negative and positive reappraisal are two emotion regulation strategies that may alleviate heartbreak in people who are upset about a romantic break-up. In this study, participants who were upset about a break-up rated infatuation, attachment, valence, and upsetness about the break-up after performing negative reappraisal of the ex-partner, positive reappraisal of the situation, and no reappraisal.

The first research question was if positive reappraisal and negative reappraisal differentially modulated love feelings. Numerically, infatuation was reported to be least intense after negative reappraisal, moderately intense after positive reappraisal, and most intense after no reappraisal. This pattern follows previous findings that negative reappraisal of the expartner, the relationship, or the future decreased feelings of infatuation (Langeslag \& Van Strien, 2016). This pattern is also in line with our hypothesis that infatuation would be lower in the negative reappraisal condition than the positive reappraisal condition. However, the omnibus test was not significant, so we found no support for our hypotheses.

Similarly, attachment was reported to be least intense after negative reappraisal, moderately intense after positive reappraisal, and most intense after no reappraisal. This pattern is in line with previous findings that negative reappraisal of the ex-partner, the relationship, or the future decreased feelings of attachment (Langeslag \& Sanchez, 2018; Langeslag \& Van Strien, 2016). This pattern also corresponds with our hypothesis that attachment would be lower in the negative reappraisal condition than the positive reappraisal condition. However, the omnibus test only trended towards significance, so we found no strong support for our hypothesis.

The second research question was if negative reappraisal and positive reappraisal differentially modulated valence of affect. Numerically, participants reported to feel least pleasant after negative reappraisal of the ex-partner, followed by the no reappraisal condition, and most pleasant after positive reappraisal of the situation. This pattern corresponds with previous findings that negative reappraisal of the ex-partner makes people feel more unpleasant than no regulation (Langeslag \& Sanchez, 2018; Langeslag \& Van Strien, 2016) and with previous findings that positive reappraisal makes people feel more pleasant (Kanske et al., 2011; Ochsner et al., 2004; Sears et al., 2003). While this pattern is also in line with our hypothesis that negative reappraisal of the ex-partner would make people feel more unpleasant than positive reappraisal of the situation, the omnibus test only trended towards significance. Therefore, we found no strong support for our hypothesis.

The third research question was if negative and positive reappraisal differentially modulate how upset people feel about a romantic break-up. In previous studies, remaining love feelings for the ex-partner were associated with reduced recovery from the break-up (Mason et al., 2012; Sbarra \& Ferrer, 2006). In a previous study, IAS infatuation and attachment scores were significantly correlated with upsetness about the break-up (Langeslag \& Sanchez, 2018). In the current study, the IAS attachment score was not correlated with upsetness about the break-up, but the IAS infatuation score tended to positively correlate with upsetness about the breakup. The ratings of upsetness about the break-up, however, were similar between conditions in the regulation task. So, the 
hypothesis that negative reappraisal of the ex-partner would make people feel less upset about the break-up than positive reappraisal of the situation was not supported by the data.

The fourth research question was if negative and positive reappraisal differentially modulate motivated attention for the ex-partner, as indicated by the LPP amplitude. Numerically, the LPP in response to the ex-partner was smallest after negative reappraisal of the ex-partner, intermediate after positive reappraisal of the situation, and largest after no reappraisal, but the omnibus tests were not significant. In an exploratory analysis, however, there was a significant difference in LPP amplitude at electrode Pz between negative reappraisal of the ex-partner and no reappraisal, suggesting that negative reappraisal decreases motivated attention to pictures of the ex-partner compared to no reappraisal. This is in line with previous findings that cognitive reappraisal reduces the LPP amplitude in response to an emotionally salient stimulus (Hajcak \& Nieuwenhuis, 2006) and that negative reappraisal of the ex-partner reduces the LPP amplitude in response to pictures of the ex-partner (Langeslag \& Sanchez, 2018). However, as this was not a preregistered analysis, we cannot draw any strong conclusions.

One feature of this study that differed from the previous study (Langeslag \& Sanchez, 2018) is that that study had an event-related design, while the current study had blocked design. The rationale for the current design was to increase power by allowing the regulation effects to accumulate over multiple trials throughout a block. However, the previous study may have had greater power because ratings were completed after every trial and then averaged, leading to an increased signal-to-noise ratio. Another limitation of this study is that we randomized the order of the blocks, which may have caused carry over effects from the regulation condition to the no reappraisal condition, which may in turn have reduced the difference between regulation conditions. However, if we would have presented the no reappraisal condition first, then ERP old/new effects (i.e., a more positive ERP for pictures that are presented for the second (and third) time than for the first time) (Rugg \& Allan, 2000) would have confounded the regulation effects on the LPP. Third, no individual differences related to gender, relationship status, and relationship duration were observed in the regulation effects on the infatuation, attachment, valence, and upsetness ratings, but a larger sample would be needed to draw more definitive conclusions about individual differences. Fourth, the participants were young adults in the greater St. Louis area, which raises the question how the results would generalize to other age groups and geographical areas. It is important to note though that we recruited from the community rather than only from university students, which increases the generalizability of the findings. Finally, several of the omnibus tests in this study approached statistical significance but were not statistically significant by our preregistered definition. So, while we cannot say anything confirmatory, we can infer from the effect sizes that positive and negative reappraisal may influence attachment, valence of affect, and the LPP amplitude. Power of the study may have been limited because of the smaller sample size. Although the sample size was determined a priori and based on a previous study (Langeslag \& Sanchez, 2018), larger sample sizes may be needed in future studies.

To conclude, this preregistered study did not find support for our hypotheses that negative reappraisal of the ex-partner and positive reappraisal of the situation differed in their effects on infatuation, attachment, valence, upsetness about the breakup, and motivated attention for the ex-partner in the short-term. Future research could use larger samples and also test the longterm effects of negative reappraisal of the ex-partner and positive reappraisal of the situation in terms of getting over break-ups.

\section{Acknowledgements \\ This work was supported by the University of Missouri Research Board. We also thank Suzanne Welcome for her input.}

\section{References}

Bryden, M. P. (1985). Laterality: Functional asymmetry in the intact brain. Canadian Psychology, 26(3), 231-235. https://doi.org/10.1037/h0084437

Compton, R. (2003). The Interface Between Emotion and Attention: A Review of Evidence from Psychology and Neuroscience. Behavioral and Cognitive Neuroscience Reviews, 2(2), 115-129. https://doi.org/10.1177/1534582303002002003

Field, T. (2011). Romantic Breakups, Heartbreak and Bereavement-Romantic Breakups. Psychology, 02(04), 382-387. https://doi.org/10.4236/psych.2011.24060

Fisher, H. E. (1998). Lust, Attaction, and Attachment in Mammalian Reproduction. Human Nature, 9(1), 2352. https://doi.org/10.1007/s12110-998-1010-5

Giuliani, N. R., McRae, K., \& Gross, J. J. (2008). The Upand Down-Regulation of Amusement: Experiential, Behavioral, and Autonomic Consequences. Emotion, 8(5), 714-719. https://doi.org/10.1037/a0013236

Gratton, G., Coles, M. G., \& Donchin, E. (1983). A new method for off-line removal of ocular artifact. Electroencephalogr Clin Neurophysiol, 55(4), 468484.

Gross, J. J. (1998). The emerging field of emotion regulation: An integrative review. Review of General Psychology, 2(3), 271-299.

Gross, J. J. (2002). Emotion regulation: Affective, cognitive, and social consequences. Psychophysiology, 39, 281291. https://doi.org/10.1002/9780470177334.ch3

Hajcak, G., Macnamara, A., \& Olvet, D. M. (2010). Eventrelated potentials, emotion, and emotion regulation: An integrative review. Developmental Neuropsychology, 35(2), 129-155. https://doi.org/10.1080/87565640903526504

Hajcak, G., \& Nieuwenhuis, S. (2006). Reappraisal modulates the electrocortical response to unpleasant pictures. Cognitive, Affective, \& Behavioral Neuroscience, 6(4), 291-297. Retrieved from https://link-springercom.ezproxy.derby.ac.uk/content/pdf/10.3758/CABN.6 .4 .291

Hajcak, G., Weinberg, A., Macnamara, A., \& Foti, D. (2011). ERPs and the study of emotion. In S.J. Luck \& E.S. Kappenman. The Oxford handbook of event-related potential components (pp. 441-472).

Kanske, P., Heissler, J., Schönfelder, S., Bongers, A., \& Wessa, M. (2011). How to regulate emotion? Neural networks for reappraisal and distraction. Cerebral 
Research Article (2019)

Cortex, 21(6), 1379-1388. https://doi.org/10.1093/cercor/bhq216

Langeslag, S. J. E., Jansma, B. M., Franken, I. H. A., \& Van Strien, J. W. (2007). Event-related potential responses to love-related facial stimuli. Biological Psychology, 76(1-2), 109-115. https://doi.org/10.1016/j.biopsycho.2007.06.007

Langeslag, S. J. E., Muris, P., \& Franken, I. H. A. (2013). Measuring romantic love: Psychometric properties of the infatuation and attachment scales. Journal of Sex Research, 50(8), 739-747. https://doi.org/10.1080/00224499.2012.714011

Langeslag, S. J. E., \& Sanchez, M. E. (2018). Downregulation of love feelings after a romantic break-up: Self-report and electrophysiological data. Journal of Experimental Psychology: General, 147(5), 720-733. https://doi.org/10.1037/xge0000360

Langeslag, S. J. E., \& Van Strien, J. W. (2016). Regulation of romantic love feelings: Preconceptions, strategies, and feasibility. PLoS ONE, 11(8), 1-29. https://doi.org/10.1371/journal.pone.0161087

Mason, A. E., Law, R. W., Bryan, A. E. B., Portley, R. M., \& Sbarra, D. A. (2012). Facing a breakup:

Electromyographic responses moderate self- concept recovery following a romantic separation. Personal Relationships, 19(3), 551-568. https://doi.org/10.1111/j.1475-6811.2011.01378.x.

Moran, T. P., Jendrusina, A. A., \& Moser, J. S. (2013). The psychometric properties of the late positive potential during emotion processing and regulation. Brain Research, 1516, 66-75. https://doi.org/10.1016/j.brainres.2013.04.018

Nezlek, J. B., \& Kuppens, P. (2008). Regulating positive and negative emotions in daily life. Journal of Personality, 76(3), 561-580. https://doi.org/10.1111/j.14676494.2008.00496.x

Ochsner, K. N., Ray, R. D., Cooper, J. C., Robertson, E. R., Chopra, S., Gabrieli, J. D. E., \& Gross, J. J. (2004). For better or for worse: Neural systems supporting the cognitive down- and up-regulation of negative emotion. NeuroImage, 23(2), 483-499. https://doi.org/10.1016/j.neuroimage.2004.06.030

Rugg, M. D., \& Allan, K. (2000). Memory retrieval: An electrophysiological perspective. In M. S. Gazzaniga (Ed.), The New Cognitive Neurosciences (pp. 805816). Cambridge: The MIT Press.

Sbarra, D. A., \& Ferrer, E. (2006). The structure and process of emotional experience following nonmarital relationship dissolution: Dynamic factor analyses of love, anger, and sadness. Emotion, 6(2), 224-238. https://doi.org/10.1037/1528-3542.6.2.224

Schupp, H. T., Flaisch, T., Stockburger, J., \& Junghöfer, M. (2006). Emotion and attention: event-related brain potential studies. Progress in Brain Research, 156, 3151. https://doi.org/10.1016/S0079-6123(06)56002-9

Sears, S. R., Stanton, A. L., \& Danoff-Burg, S. (2003). The yellow brick road and the Emerald City: Benefit finding, positive reappraisal coping, and posttraumatic growth in women with early-stage breast cancer. Health Psychology, 22(5), 487-497. https://doi.org/10.1037/0278-6133.22.5.487

Slotter, E. B., Gardner, W. L., \& Finkel, E. J. (2010). Who am i without you? the influence of romantic breakup on the self-concept. Personality and Social Psychology Bulletin, 36(2), 147-160. https://doi.org/10.1177/0146167209352250 